\title{
An Effective and Trustable Spatial Service Recommendation Algorithm for Spatial Query Retrieval in Geo-Social Network
}

\author{
K. Lakshmaiah ${ }^{1}$, S. Murali Krishna ${ }^{2}$, B. Eswara Reddy ${ }^{3}$ \\ ${ }^{1}$ awaharlal Nehru Technological University, Hyderabad, India \\ ${ }^{2} \mathrm{~S}$ V College of Engineering, Karakambadi Road, Tirupati, India \\ ${ }^{3}$ Dept. of Computer Science \& Engineering, JNTUA College of Engineering, Kalikiri-517234, Chittoor (Dt), India
}

\begin{tabular}{l} 
Article Info \\
\hline Article history: \\
Received Jul 29, 2018 \\
Revised Sep 10, 2018 \\
Accepted Sep 24, 2018 \\
\hline
\end{tabular}

\section{Keywords:}

Effective and trustable spatial service recommendation framework

Geo-Social network

Information retrieval

Precision

Query retrieval time

Query Retrieval Time (QRT)

Recall

Spatial query

\begin{abstract}
The spatial information (e.g., restaurants/hotels) is related with the keyword(s) to indicate their businesses, services and features. The main issue of relevant information retrieval is to query an entity which includes a set of spatial query keywords and have the smallest amount of inter-object distance. The spatial queries with keywords have not been extensively explored. Still, the traditional method was focused on the multidimensional data. Previous works mostly targeted to predict the top-k Nearest Neighbors keyword query, where every keyword should be equivalent to the whole querying keywords. However, the mechanism does not consider the density of data entities in the spatial space. To overcome the above issues, An Effective and Trustable Spatial Service Recommendation (ETSSR) algorithm focuses on the most relevant information retrieval with the enhanced accuracy and minimal retrieval time for spatial information services. The main goal of work is to provide best spatial information retrieval with an accurateness of location prediction and minimal information retrieval time. The system minimizes the classification issue and visualization problem for spatial information in Geospatial Social network. The system improves the spatial information retrieval with an accuracy of location prediction and minimizes the information retrieval time compare than existing methods. Based on Experimental estimations, proposed ETSSR+KNN enhanced $0.48 \mathrm{P}$ (Precision) and $0.49 \mathrm{R}$ (Recall) and minimized 28 milliseconds query retrieval time.
\end{abstract}

Copyright $\odot 2018$ Institute of Advanced Engineering and Science. All rights reserved.

\section{Corresponding Author:}

Lakshmaiah K,

Research Scholar,

Jawaharlal Nehru Technological University,

Hyderabad, India.

Email: lakshmaiah.jntu@gmail.com

\section{INTRODUCTION}

The spatial databases (e.g., restaurants/hotels) are associated with the keyword(s) to indicate their businesses/services/features. An interesting issue known as Keyword related information retrieval is to query an object which contains a set of query keywords and have the minimum inter-objects distance. Spatial queries with keywords have not been extensively explored. Still, the methods were focused on the multidimensional data. Existing works mainly targeted to find top-k Nearest Neighbors query, where each node should be equivalent to the whole querying keywords. However, the technique does not consider the density of data objects in the spatial space. 


\subsection{Problem}

Based on the literature study, the research work observed that the increasing availability and importance of keyword rating in spatial query evaluation for the relevant spatial information retrieval. The method can facilitate people travel not only near areas but also in city that is new for users. For instance, the visitor arrangement to visit a city requires to shopping, dining and accommodation. It is popular of all these needs can be satisfied with best information accuracy and minimal time without long distance travelling. Current system utilizes the multidimensional indexes for top-k query retrieval. Initially, it splits the high dimensional spaces and involves an arbitrary set of user-specified attributes. However, the method is not flexible for all possible attribute combinations. Here, there one more challengeable tasks are to receive different type's rankings for combined attributes. However, the method does not assure the accuracy of relevant information ranking. These methods have low efficiency for the incremental query.

\subsection{Background}

In [1] focused on large-scale recommender frameworks which were taken benefit of the features of the underlying in the social network. The method focused on the variety and unpredictability of the social connections. The technique tackled the issues of data size and communication speeds in social graphs and tested the scalability of conventional recommender frameworks. In [2] described a systematic mechanism to identify possible spatiotemporal prototypes of tasks by determining the challenges through numerous interconnected methods: utilizing kernel density evaluation for smoothed social media intensity surfaces; using event-unconnected social media posts to support map relation task occurrence, and regularizing task indicators depends on historical variation. In [3] introduced a graph investigation based strategy which studied social networks with geographic data and differentiated geographic distance influences in social structure. In [4] explained two protocols for offering complete confidentiality to concern the SP (Service Provider), and convenient confidentiality with respective clients. In [5] analyzed the geo-social correlations among LBSN (Location Based Service Network) clients at the task level. The unified influence metric, namely global iteration (GI) and dynamic neighborhood expansion (DNE) worked to estimate client influence with tight hypothetical issue bounds in capturing the geo-social closeness of LBSN clients. In [6] addressed the socio-spatial graph which depends on life prototypes, where clients attached to geographical objects utilizing life-pattern edges. The strategy considered two implementations of sociospatial graph storage. The implementation utilized a relational database for a variety of queries and dataset.

In [7] studied the complexity of GeoSN clients to manage the contributed information. The strategy addressed two confidentiality hazards that occurred in GeoSNs: location confidentiality and absence confidentiality. In [8] assured for finding the Circle of Friends (CoF) size k were an NP-hard issue. The mechanism assured to offer a group of friends with diameter. In [9] evaluated many kinds of functions for describing the price and numerous methods solved the CoSKQ issue. The incorporated price function contained all existing price functions as special cases, and the unified method resolved the CoSKQ issue with the unified price function. In [10] introduced Social-aware top-k Spatial Keyword (SkSK) query and spatial keyword query. Social relevance feature utilized to enhance the semantics of the conventional spatial keyword query. In [11] expressed correct and estimated solutions when the number $\kappa$ of query keywords is small. However, when $\kappa$ increases, it becomes costly. In [12] designed a Spatial-aware Interest Group (SIG) query that retrieved a client group of size $\mathrm{k}$ where every client interested in the query keywords. It closed to the Euclidean space. In [13] surveyed some kinds of geo-textual indexes. It differentiated the spatial-keyword query performance. In [14] designed a Spatial Inverted Index (SII) which enhanced the performance of top-k spatial keyword queries. The index mapped a set of entities in every separate term. The entities were stored in a different manner such as the filing frequency of the term and retrieved in minimizing the keyword relevance and spatial proximity.

In [15] expressed the privacy protection to guarantee the effectiveness of KNN query processing. The framework managed Wise Dummy Selection Location (WDSL) algorithm to ensure the location confidentiality. In [16] studied an efficiently processing of always moving top-K spatial keyword (MkSK) queries over spatial keyword information. The mechanism guarantee the authority of reported outcomes offering the customer stays within a region. In [17] explained integration of Adaptive Weight Ranking Policy (AWRP) with intelligent classifiers (NB-AWRP-DA and J48-AWRP-DA) via dynamic aging factor to improve classifiers power of prediction. The methods are used to choose the best subset of features. In [18] introduced a new framework called Fuzzy based contextual recommendation system for classification of customer reviews. It extracts the information from the reviews based on the context given by users. In [19] studied to identify the best classifiers for class imbalanced health datasets through a cost-based comparison of classifier performance. The unequal misclassification costs were represented in a cost matrix, and cost-benefit. In [20] described a location based and preference-aware recommender system to offers a specific user a set of spatial information like restaurants and shopping malls in certain geospatial range. 


\subsection{Objectives:}

The paper objectives are following as:

a) To design an Effective and Trustable Spatial Service Recommendation algorithm for retrieving the most relevant spatial information with the best accuracy and minimal time in Geo-Social Network.

b) To apply similarity function to determine similar users with their interest in spatial information retrieval for offering best spatial information retrieval with an accuracy of location prediction.

c) To reduce the prediction issues to visualize the relevant information in Geospatial Social network.

d) To improve the spatial information retrieval with an accuracy of location prediction and minimize the information retrieval time compare than existing methods.

\section{METHOD}

The design describes the complete architecture for spatial query relevant information retrieval with the best accuracy and minimal retrieval time in Geo-social Network. Figure 1 explores the processing steps of proposed work in details. Here, the design explores the pre-processing steps, involved GSN (Geo-social Network) authority and algorithm details with pseudo code. The system also works to predict the query location based closeness of users. The techniques dedicated to offer trustable and reliable spatial query service in GSN. To offer the best solution for above problem, an Effective and Trustable Spatial Service Recommendation algorithm introduced to offer most relevant information retrieval with the best accuracy and retrieval minimal time for spatial information services. The main goal of a research study is to offer best spatial information retrieval with an accuracy of location prediction and minimal information retrieval time. The technique also works to retrieve most relevance information, based on the user query, not the popularity of information or search engine optimization (SEO) based services. The technique also works to rank the spatial information based on query demand. The system utilizes a 'similarity function' to determine similar users with their interest in spatial information retrieval. The objects connected with the principal query keyword objects are to retrieve the relevant information. The system reduces the classification problem to visualize the relevant information in Geospatial Social network. The principal object also helps to proposed framework to predict the most relevant and highly reliable spatial information for users. The spatial information services can find all restaurants in a given area; where nearest neighbour retrieval can only discover the restaurant closest to a given address. The proposed techniques utilized two types of concept for of ranking services like service recommendation and decision support. The technique predicts the ranking of spatial information based on user location. The proposed method utilizes combined object rankings of attributes which are collected from different sources. The method minimizes multiple time input raking issues to predict the most relevant information.

\subsection{User}

If, a user need to retrieving the information which is already stored in a centralized storage server. First, user should register their details into storage server. These details are maintained in a centralized Database. Once user registered their details, then they can search their query in centralized storage server.

\subsection{GSN (Geo-Social Network) Authority}

The GSN authority can view the user details and upload the spatial information with descriptions. Here, GSN authority can also alter about spatial information whatever changes and whatever information he/she required. It provides a chance for advertisers to get a huge set of spatial information, which could potentially lead to identifying a user interest.

\subsection{Spatial Dataset}

The spatial-keyword search has obtained substantial attention from research society. Some previous frameworks concentrated for retrieving individual entities by identifying a query comprising of a query location and a set of query keywords. Every retrieved entity is related to keywords relevant to the spatial query keyword information and it is closest to the spatial query. The similarity between files is applied to compute the relevance of two sets of keywords. As it is probably no individual entity is related to all query keywords. Some additional works aim to retrieve many entities which together cover all query keywords. While potentially a huge amount of entity integrations fulfill the requirement, the research issue is that the retrieved entities must have a popular spatial data connection. The researcher put forward the issue to retrieve entities which cover all spatial query keywords, have the smallest amount of inter-entities distance and closed to a spatial query location. 


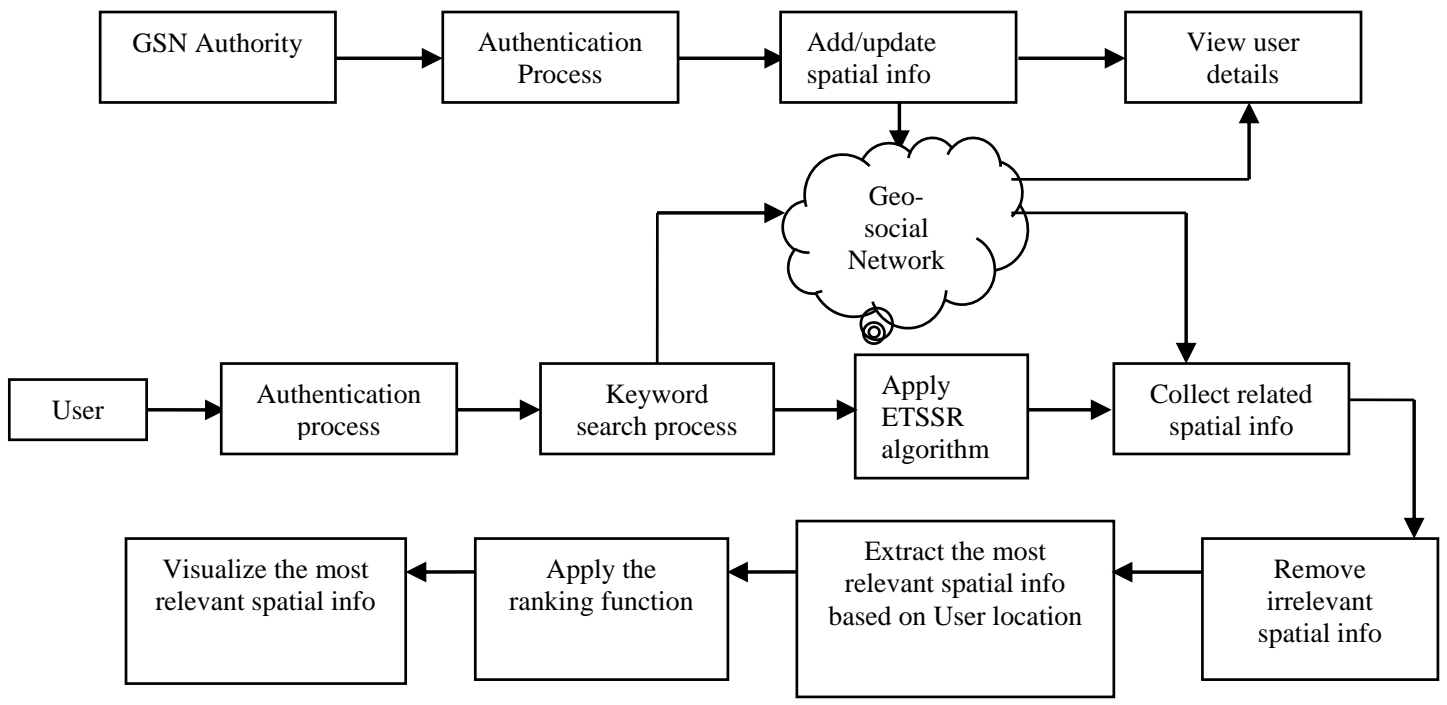

Figure 1. Workflow diagram of Architecture Diagram of Proposed System

\subsection{Keyword Search}

Specified a spatial database every entity related with one or more spatial keyword information. An entity with numerous spatial keywords is changed to numerous entities placed at the same location without loss of generalization with a single distinct spatial keyword. An ETSSR method manufactures less new candidate keyword covers when processing a candidate keyword cover. The ETSSR algorithm also processed amount of candidate keyword covers is optimal the amount of keyword covers created. Consecutively, it concludes that the amount of keyword covers created in the ETSSR algorithm. The summary is independent of main query keyword as the investigation does not apply any parameter on the selection method main query keyword.

\subsection{User Point of Interests}

The objective of the interface is to offer a point of interest information with, at least, a location, some compulsory features and optional descriptions. It is offering the spatial data; the element that develops the interface utilizes the map location database information to locate and exhibit a point of interest (POI) or to select POI as route waypoint. The element does not only offer spatial data search functionalities for the local database but also expresses, how to relate external keyword search technique to the element. The method also improves the keyword search criteria and the list of extracted outcomes. The proposed design also introduces a resolution to acquire convention POIs or to modify content and explanation of local POI dynamically.

\subsection{Effective and Trustable Spatial Service Recommendation Algorithm}

An Effective and Trustable Spatial Service Recommendation algorithm introduced to focus on the most relevant information retrieval with the best accuracy and minimal retrieval time for spatial information services. The mechanism works to retrieve most relevance information, depends on the user query, not the popularity of information or search engine optimization (SEO) based services. The framework utilizes a 'similarity function' to determine similar users with their interest in spatial query information retrieval. The entities related to the main query keyword entities are to retrieve the relevant information. The principal entity also assists to the proposed framework to compute the most relevant and highly reliable spatial information for users.

The spatial information services can locate all restaurants, hospital and bank in a given area; where nearest neighbor retrieval can only discover the restaurant, hospital and bank closest to a provided address. Here, Geo-social Network (GSN) authority can add and update the query information (QI) frequently. GSN can view the list of registered GSN user. User can enter spatial query (SQ) based their requirement. Next system predicts user location accuracy (LA) to recommend the spatial query information services (SQIS). The design removes the irrelevant information from collected information source (IS). The mechanism process the query to get the most relevant information (RI) from GSN. Proposed techniques used two types of ranking function like service recommendation and decision support technique to offer most RI. The proposed methodology utilizes integrated entity rankings of features which are gathered from 
different sources. The technique reduces multiple time input ranking issues to predict the most relevant information. The pseudo code Effective and Trustable Spatial Service Recommendation (ETSSR) Algorithm is explained below in details:

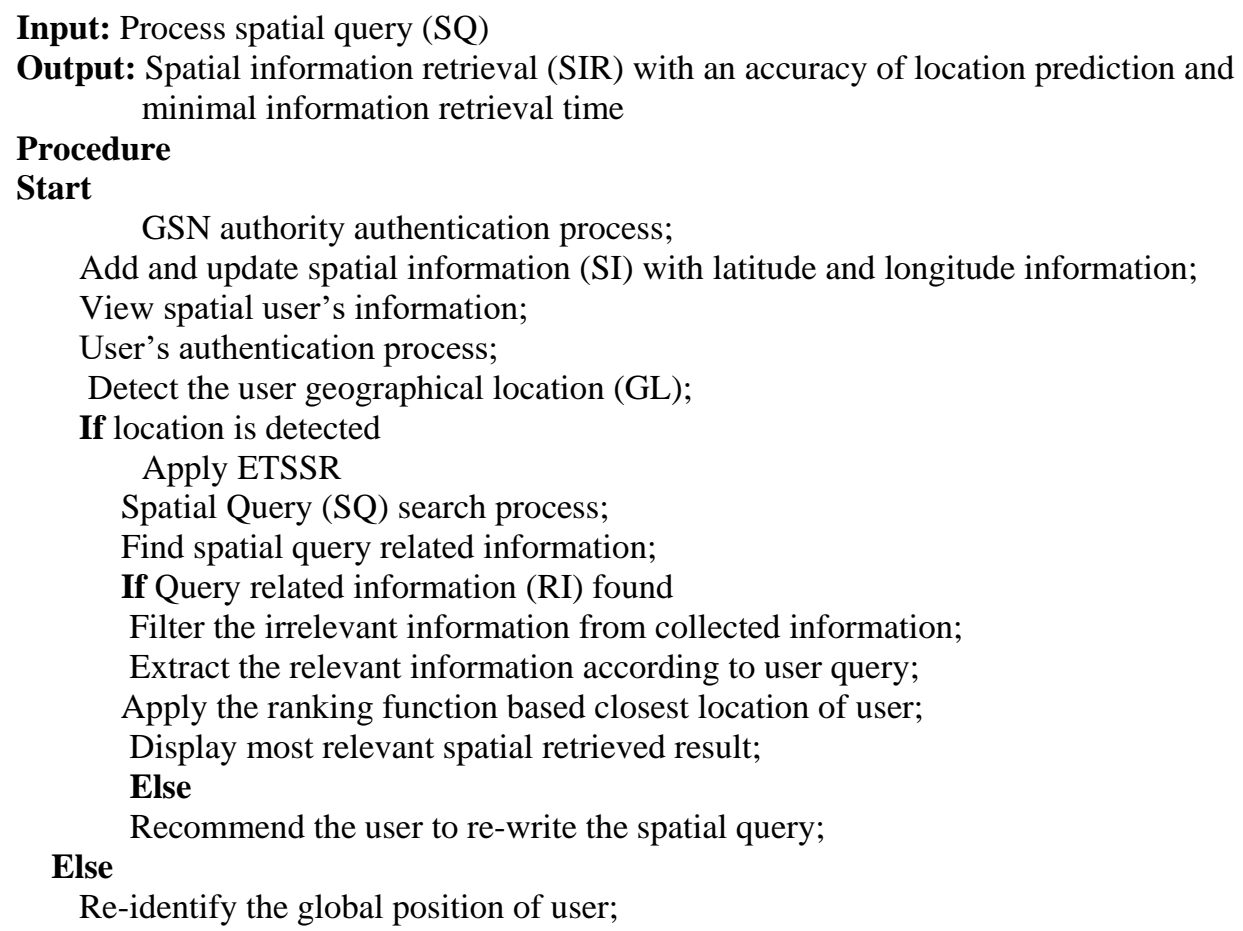

\section{RESULTS AND DISCUSSION}

\subsection{Experiments Setup}

The proposed mechanism is deployed with Intel Core $i^{5} 7^{\text {th }}$ Generation Processor with 10 GB RAM, 400 GB Memory and Windows7 professional operating system. The proposed mechanism is developed in Java programming with JDK (Java Development Kit) 1.8, Netbeans 8.0 Integrated Development Environment, Apache Tomcat 8.0.3, and MYSQL 5.5 database.

\subsubsection{Data}

For experimental evaluations, proposed mechanism select spatial domain three different datasets namely Hospital dataset with 250 records, Restaurant dataset 200 records and Bank dataset with 250 records is collected with name, location, and services descriptions details.

\subsection{Simulation Result}

In the section, an Effective \& Trustable Spatial Service Recommendation (ETSSR) algorithm investigates the mathematical structure of the relevant information retrieval with the best accuracy like precision $(\mathrm{P})$, recall( $\mathrm{R})$ and retrieval minimal time for spatial information services in Geo-social Network. Here, it illustrates precision, recall, and query retrieval time (QRT) to compute the performance of proposed ETSSR method. It identified, how can achieve the best accuracy with minimal query retrieval time for spatial information retrieval in Geo-Social Network (GSN).

\subsubsection{Precision (P)}

The precision explain the agreement of a set of retrieved results among themselves. Precision represents the deviation of set of retrieved results from the arithmetic mean of the set. The precision concentrated on the identification and elimination of systematic errors. Precision can be computed as in Equation (1).

$$
\text { Precision }=\frac{T_{P}}{T_{P}+F_{P}}
$$

Where, $T_{P}$ is truly positive and $F_{P}$ is a false positive. 


\subsubsection{Recall}

Recall expressed as the total number of relevant information which is extracted based on search queries and divided by the total number of available relevant information. Recall is the ratio of the total amount of relevant searched information to the total number available relevant records in centralized database. It is described in Equation (2).

$$
\operatorname{Re} \text { call }=\frac{T_{p}}{T_{P}+F_{N}}
$$

Where, $T_{P}$ is truly positive and $F_{N}$ is a false negative.

\subsubsection{Query Retrieval Time (QRT)}

The proposed ETSSR method illustrates a mathematical model for query retrieval time in Equation (3). The proposed technique is computed query retrieval time based on time taken by centralized server to process the user requested query. Query Retrieval Time (QRT) is calculated as:

$$
Q R T=T_{C D} \times T_{A R}
$$

Where, $\mathrm{T}_{\mathrm{CD}}$ is a total number of spatial record and $\mathrm{T}_{\mathrm{AR}}$ is average retrieval time for a query processing of spatial user.

Table 1 illustrates the precision $(\mathrm{P})$, recall(R) and Query Retrieval Time (QRT) in milliseconds for Hospital, Restaurant and Bank spatial dataset. The proposed ETSSR method demonstrates their average values for respective constraint with the respective dataset. Here, proposed Effective \& Trustable Spatial Service Recommendation (ETSSR) algorithm is estimated with previous methodologies such as Most Preferred Category based Recommendation (MPCR) [20], Preference-based Collaborative Filtering (PCF) [20] and Location-based Collaborative Filtering (LCF) [20] mechanisms which results are displayed in Table 1.

Table shows the precision; recall and query retrieval time for Hospital, Bank and Restaurant spatial datasets. According to table 1 outcomes, it noticed ETSSR performed well on Hospital, Bank and Restaurant spatial dataset. Finally, the article claims that the proposed ETSSR is the best approach for overall spatial datasets. The technique is integrated with KNN (K-Nearest Neighbor) classifier to predict to retrieve query location and user accuracy.

Table 1. Precision (P), Recall (R), and Query Retrieval Time (QRT) in milliseconds for Hospital, Restaurant

\begin{tabular}{|c|c|c|c|c|c|c|c|c|c|}
\hline \multirow[t]{2}{*}{ Learning Algorithms } & \multicolumn{3}{|c|}{ Hospital } & \multicolumn{3}{|c|}{ Restaurant } & \multicolumn{3}{|c|}{ Bank } \\
\hline & $\mathrm{P}$ & $\mathrm{R}$ & QRT & $\mathrm{P}$ & $\mathrm{R}$ & QRT & $\mathrm{P}$ & $\mathrm{R}$ & QRT \\
\hline MPCR & 0.39 & 0.29 & 190 & 0.5 & 0.15 & 250 & 0.28 & 0.5 & 40 \\
\hline PCF & 0.43 & 0.41 & 620 & 0.6 & 0.24 & 750 & 0.35 & 0.7 & 110 \\
\hline LCF & 0.19 & 0.13 & 50 & 0.2 & 0.1 & 110 & 0.21 & 0.15 & 30 \\
\hline ETSSR & 0.99 & 0.98 & 42 & 0.88 & 0.91 & 50 & 0.97 & 0.95 & 15 \\
\hline
\end{tabular}
and Bank spatial dataset

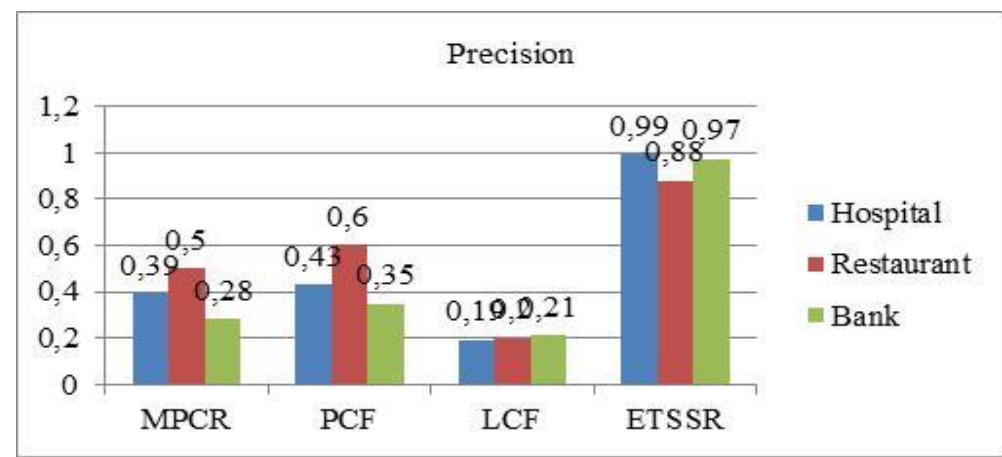

Figure 2. Precision for Hospital, Restaurant and Bank dataset 


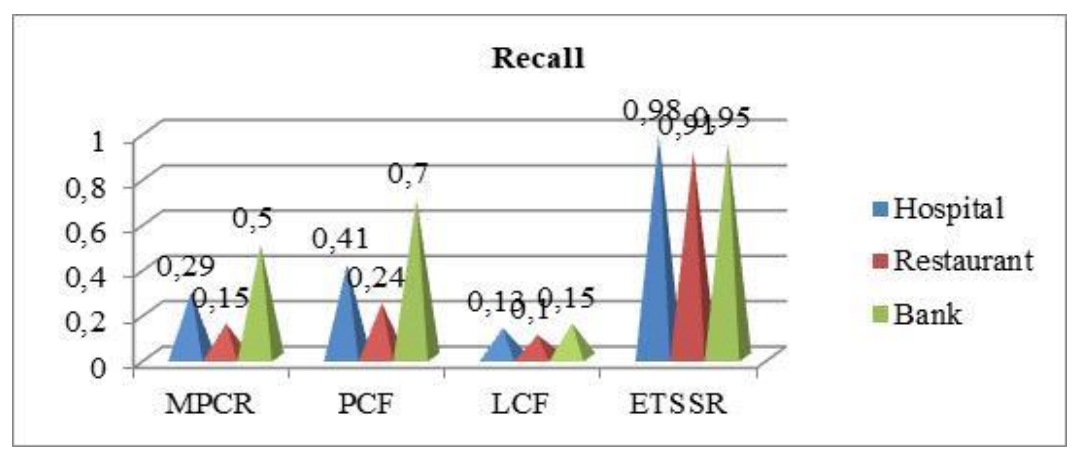

Figure 3. Recall for Hospital, Restaurant and Bank dataset

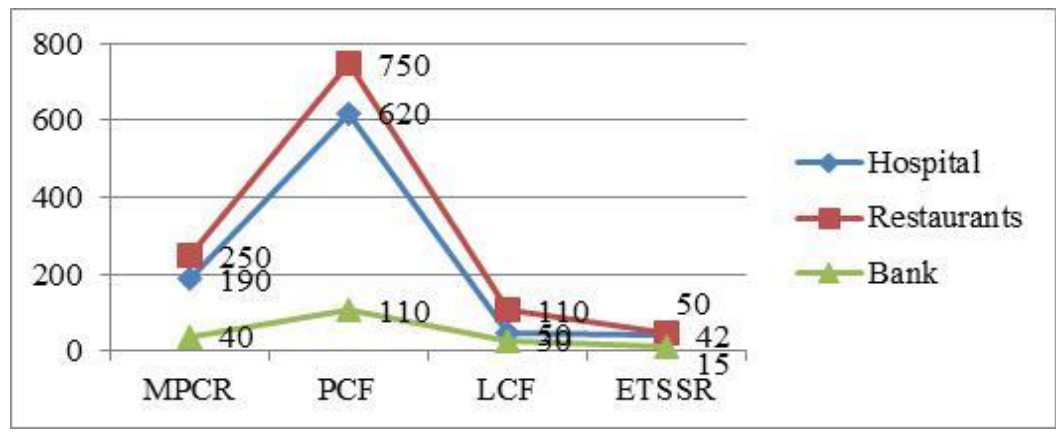

Figure 4. Query Retrieval Time for Hospital, Restaurant and Bank dataset

According to Figure 2 to 4 observations for Hospital, Restaurant and Bank spatial datasets, the proposed ETSSR+KNN approach computes precision, recall and query processing time for identifying the effectiveness of technique. The proposed ETSSR+KNN is computed with MPCR, PCF and LCF existing approach behalf of precision, recall and query processing time. PCF is the nearest competitor on precision and recall constraints. The method is utilized to retrieve web based query information. However, it fails to predict and categorized the retrieved query of multiple type information from different sources. LCF is the closest method behalf of on query retrieval time (QRT) constraint. LCF method is used to apply ranking function to retrieve relevant result first from multiple type of query information database. However, the technique consume more time for query retrieval with less location prediction accuracy. The proposed ETSSR+KNN provide enhanced $0.48 \mathrm{P}$ (Precision) and $0.49 \mathrm{R}$ (Recall) and minimized 28 milliseconds query retrieval time. Finally, the paper announces the proposed ETSSR+KNN approach performs best on every parameter \& respective input constraints.

\section{CONCLUSION}

An Effective and Trustable Spatial Service Recommendation Framework is presented to consternate on the most relevant information retrieval with the accurateness and minimal retrieval time for spatial information services. The major aim of technique is to offer best spatial information retrieval with location prediction accuracy and minimal information retrieval time. In Geospatial Social network, the classification and visualization issues are minimized for the spatial query relevant information retrieval. The proposed framework is highly dedicated to offer most relevant information to users. The spatial information services can locate all restaurants, hospital and bank related information in a given area; where nearest neighbour retrieval can only discover the hospital and bank related information, closest to a given address. Two kinds of concepts utilized to the proposed framework such as ranking services like service recommendation and decision support. The technique predicts the ranking of spatial information depends on user location. The proposed ETSSR+KNN provide enhanced $0.48 \mathrm{P}$ (Precision) and $0.49 \mathrm{R}$ (Recall) and minimized 28 milliseconds query retrieval time. Finally, the paper announces the proposed ETSSR approach performs best on every parameter $\&$ respective input constraints. 
In future, the work can be extended to process spatial query information in cloud environment with data and location privacy because; security is challengeable task in Geo-Social Network during data contribution and retrieval.

\section{REFERENCES}

[1] Eirinaki, M., Gao, J., Varlamis, I., \& Tserpes, K., "Recommender Systems for Large-Scale Social Networks: A review of challenges and solutions", Future Generation Computer Systems, vol. 78, pp. 413-418, 2018.

[2] Gao, Y., Wang, S., Padmanabhan, A., Yin, J., \& Cao, G., "Mapping spatiotemporal patterns of events using social media: a case study of influenza trends", International Journal of Geographical Information Science, vol. 32, no. 3, pp. 425-449, 2018.

[3] Scellato, S., Mascolo, C., Musolesi, M., \& Latora, V., "Distance Matters: Geo-social Metrics for Online Social Networks", In WOSN, pp. 1-9, 2010.

[4] Mascetti, S., Freni, D., Bettini, C., Wang, X. S., \& Jajodia, S., "Privacy in geo-social networks: proximity notification with untrusted service providers and curious buddies", The VLDB Journal—the International Journal on Very Large Data Bases, vol. 20, no. 4, pp. 541-566, 2011.

[5] Zhang, C., Shou, L., Chen, K., Chen, G., \& Bei, Y., "Evaluating geo-social influence in location-based social networks", In Proceedings of the 21st ACM international conference on Information and knowledge management, ACM, pp.1442-1451, 2012.

[6] Doytsher, Y., Galon, B., \& Kanza, Y., "Querying geo-social data by bridging spatial networks and social networks", In Proceedings of the 2nd ACM SIGSPATIAL International Workshop on Location Based Social Networks, pp. 39-46, 2010.

[7] Freni, D., Ruiz, C. V., Mascetti, S., Bettini, C., \& Jensen, C. S., "Preserving location and absence privacy in geosocial networks", In Proceedings of the 19th ACM international conference on Information and knowledge management, pp. 309-318, ACM, 2010.

[8] Liu, W., Sun, W., Chen, C., Huang, Y., Jing, Y., \& Chen, K., "Circle of friend query in geo-social networks", In International Conference on Database Systems for Advanced Applications, pp. 126-137, 2012.

[9] H. K. H. Chan, C. Long and R. C. W. Wong, "On Generalizing Collective Spatial Keyword Queries", IEEE Transactions on Knowledge and Data Engineering. pp. 1-18, 2018.

[10] Wu, D., Li, Y., Choi, B., \& Xu, J., "Social-aware top-k spatial keyword search", In Mobile Data Management (MDM), 2014 IEEE 15th International Conference, vol. 1, pp. 235-244, 2014.

[11] Yao, B., Tang, M., \& Li, F., "Multi-approximate-keyword routing in GIS data", In Proceedings of the 19th ACM SIGSPATIAL International Conference on Advances in Geographic Information Systems, pp. 201-210, 2011.

[12] Li, Y., Wu, D., Xu, J., Choi, B., \& Su, W., "Spatial-aware interest group queries in location-based social networks", Data \& Knowledge Engineering, vol. 92, pp. 20-38, 2014.

[13] Chen, L., Cong, G., Jensen, C.S., \& Wu, D., "Spatial keyword query processing: an experimental evaluation" In Proceedings of the VLDB Endowment, vol. 6, no. 3, pp. 217-228, 2013.

[14] Rocha, J. B., Gkorgkas, O., Jonassen, S., \& Nørvåg, K., "Efficient processing of top-k spatial keyword queries", In International Symposium on Spatial and Temporal Databases, pp. 205-222, 2011.

[15] Alrahhal, M. S., Khemakhem, M., \& Jambi, K., "Agent-Based System for Efficient kNN Query Processing with Comprehensive Privacy Protection", (IJACSA) International Journal of Advanced Computer Science and Applications, vol. 9, no. 1, 2018.

[16] Wu, D., Yiu, M. L., Jensen, C. S., \& Cong, G., "Efficient continuously moving top-k spatial keyword query processing", In Data Engineering (ICDE), IEEE 27th International Conference, pp. 541-552, 2011.

[17] Olanrewaju, R. F., \& Azman, A. W. Intelligent Cooperative Adaptive Weight Ranking Policy via dynamic aging based on NB and J48 classifiers. Indonesian Journal of Electrical Engineering and Informatics (IJEEI), 2017; 5(4): 357-365.

[18] Sulthana, R., \& Ramasamy, S. Context Based Classification of Reviews Using Association Rule Mining, Fuzzy Logics and Ontology. Bulletin of Electrical Engineering and Informatics, 2017; 6(3): 250-255.

[19] Rao, R. R., \& Makkithaya, K. Learning from a Class Imbalanced Public Health Dataset: a Cost-based Comparison of Classifier Performance. International Journal of Electrical and Computer Engineering, 2017; 7(4): 2215-2222.

[20] Bao, J., Zheng, Y., \& Mokbel, M. F., "Location-based and preference-aware recommendation using sparse geosocial networking data", In Proceedings of the 20th international conference on advances in geographic information systems, ACM, pp. 199-208, 2012. 